\title{
Tumor Immunity and Autoimmunity Induced by Immunization with Homologous DNA
}

\author{
Lawrence W. Weber, ${ }^{\star}$ Wilbur B. Bowne, ${ }^{\S}$ Jedd D. Wolchok, ${ }^{\star \ddagger}$ Roopa Srinivasan, ${ }^{\star}$ Jie Qin, ${ }^{\star}$ Yoichi Moroi, ${ }^{\star}$ Raphael Clynes, ${ }^{\ddagger}$ \\ Ping Song, ${ }^{\star}$ Jonathan J. Lewis, ${ }^{\star \S}$ and Alan N. Houghton ${ }^{\star \ddagger}$ \\ *The Swim Across America Laboratory, Sloan-Kettering Division, Cornell University Graduate School of Medical Sciences, New York \\ 10021; ${ }^{\ddagger}$ Department of Medicine and ${ }^{\S}$ Department of Surgery, Memorial Sloan-Kettering Cancer Center; and ${ }^{\|}$The Rockefeller University, \\ New York 10021
}

\begin{abstract}
The immune system can recognize self antigens expressed by cancer cells. Differentiation antigens are prototypes of these self antigens, being expressed by cancer cells and their normal cell counterparts. The tyrosinase family proteins are well characterized differentiation antigens recognized by antibodies and $\mathrm{T}$ cells of patients with melanoma. However, immune tolerance may prevent immunity directed against these antigens. Immunity to the brown locus protein, gp75/ tyrosinase-related protein-1, was investigated in a syngeneic mouse model. C57BL/6 mice, which are tolerant to gp75, generated autoantibodies against gp75 after immunization with DNA encoding human gp75 but not syngeneic mouse gp75. Priming with human gp75 DNA broke tolerance to mouse gp75. Immunity against mouse gp75 provided significant tumor protection. Manifestations of autoimmunity were observed, characterized by coat depigmentation. Rejection of tumor challenge required $\mathrm{CD}^{+}$and $\mathrm{NK} 1.1^{+}$cells and $\mathrm{Fc}$ receptor $\gamma$-chain, but depigmentation did not require these components. Thus, immunization with homologous DNA broke tolerance against mouse gp75, possibly by providing help from $\mathrm{CD}^{+}{ }^{+} \mathrm{T}$ cells. Mechanisms required for tumor protection were not necessary for autoimmunity, demonstrating that tumor immunity can be uncoupled from autoimmune manifestations. (J. Clin. Invest. 1998. 102: 1258-1264.) Key words: melanoma • differentiation • xenogeneic $\cdot$ tolerance $\cdot$ tyrosinase
\end{abstract}

\section{Introduction}

Immune recognition of human cancer is commonly directed against differentiation antigens $(1,2)$. A differentiation antigen is expressed in a cell lineage at a distinct stage of differentiation and distinguishes one cell type or cell lineage from other cell types or lineages (3). Immunity to differentiation antigens is presumably difficult to elicit due to immune tolerance. However, T cells and B cells that potentially recognize differentiation antigens in peripheral tissues are not necessarily de-

Address correspondence to Alan N. Houghton, Memorial Sloan-Kettering Cancer Center, 1275 York Avenue, New York, NY 10021. Phone: 212-639-7595; FAX: 212-794-4352; E-mail: a-houghton@ ski.mskcc.org

Received for publication 14 May 1998 and accepted in revised form 17 July 1998

J. Clin. Invest.

(C) The American Society for Clinical Investigation, Inc. 0021-9738/98/09/1258/07 \$2.00

Volume 102, Number 6, September 1998, 1258-1264

http://www.jci.org leted from the immune repertoire during development of the immune system. This assumption implies that lymphocytes capable of reacting with differentiation antigens remain in the immune repertoire but do not typically respond to cancers expressing these antigens.

Immune recognition of differentiation antigens in humans has been best characterized in patients with melanoma. Melanocyte differentiation antigens, which are antigens expressed by melanomas and normal melanocytes at some stage of differentiation (4), are commonly recognized by the immune system of patients with melanoma (2). The best characterized melanoma differentiation antigens are the melanosomal membrane glycoproteins, including tyrosinase, gp75/tyrosinaserelated protein 1 (TRP-1), ${ }^{1}$ TRP-2, and gp100/pMel17. These gene products play important roles in cutaneous pigmentation and determine coat color of mice, e.g., albino (tyrosinase), brown (gp75), slaty (TRP-2), and silver (gp100) (5). Products of these genes can be recognized by antibodies and $\mathrm{T}$ cells, including both $\mathrm{CD}^{+}$and $\mathrm{CD}^{+} \mathrm{T}$ cells (6-16). Thus, these proteins can be recognized by multiple components of the immune system, suggesting that broad immunity to these differentiation antigens is possible.

Despite the prevalence of antibodies and $\mathrm{T}$ cells against these autoantigens in patients with melanoma, it is uncertain whether this immune recognition can translate into immunity that can actually reject the cancer, especially in the face of immune tolerance. A potential sequela of immunity against differentiation antigens is autoimmunity that leads to destruction of normal tissues. We show that tolerance to the mouse melanosomal glycoprotein gp75 can be broken by immunization with DNA encoding an evolutionarily conserved, xenogeneic gp75 protein that is highly homologous to the mouse brown locus protein. Thus, immunization with homologous DNA leads to immunity that cross-reacts with the self protein, breaking tolerance to the self protein. This immunity provides tumor protection and triggers autoimmune sequelae. The mechanisms involved in tumor protection induced by this immunization are distinct from the mechanisms involved in manifestations of autoimmunity.

\section{Methods}

Cell lines and tissue culture. B16F10/LM3 is a pigmented mouse melanoma cell line of C57BL/6 origin derived from the B16F10 line, which was kindly provided by Dr. Isaiah Fidler (M.D. Anderson Cancer Center, Houston, TX) (17). The B16F10/LM3 line was isolated after three passages as lung metastases in C57BL/6 mice (18). The mouse fibroblast cell line L929 was obtained from the American Type Culture Collection (Rockville, MD). Cell lines were grown in

1. Abbreviations used in this paper: Endo $\mathrm{H}$, Endo- $\beta-\mathrm{N}$-acetylglucosaminidase $\mathrm{H}$; NK, natural killer; TRP-1, tyrosine-related protein 1. 
minimal essential media plus $1 \%$ nonessential amino acids, $100 \mu \mathrm{g} / \mathrm{ml}$ each penicillin and streptomycin, and $2 \mathrm{mM}$ glutamine (GIBCO, Grand Island, NY) supplemented with $10 \%$ heat-inactivated FBS (Hazelton, Lenexa, KS). Cells were routinely tested for mycoplasma and were negative.

Plasmid constructions. A $1.9 \mathrm{~kb}$ EcoR1 fragment containing the full-length mouse $g p 75$ cDNA was isolated from the $p M T 4$ plasmid (kindly supplied by Dr. T. Shibahara, Tohoku University School of Medicine, Japan) (19) and subcloned into unique Not1/BamH1 sites of the WRG/BEN plasmid (20), which contains a cytomegalovirus promoter and kanamycin resistance gene. The original WRG7077 plasmid (null vector) used to construct WRG/BEN (which contains altered polylinkers) was kindly provided by PowderJect (Middleton, WI). Full-length human $g p 75$ cDNA (6) was inserted into WRG/BEN plasmid in the same manner. The plasmid encoding human gp75 was designated $h g p 75$ and the mouse $g p 75$ plasmid was designated $m g p 75$.

DNA immunization. The method for DNA immunization has been reported (20). Plasmid DNA was purified and coated onto $2.1-\mu \mathrm{m}$ gold bullets as described (20). Briefly, mice were anesthetized with Metofane inhalation (Pitman-Moore, Mundelein, IL) and hair was removed with Nair depilatory cream (Carter-Wallace, New York, NY) exposing the abdominal skin for immunization. Animals were immunized by delivering gold/DNA complexes using a helium-driven gene gun (PowderJect) into each abdominal quadrant for a total of four injections per mouse. The gene gun was operated at a helium pressure of 400 pounds psi. Each bullet contains $1 \mu \mathrm{g}$ of DNA; therefore, each mouse received $4 \mu \mathrm{g}$ of DNA per immunization. Antibodies against gp75 were detected by immunoprecipitation as described below.

Mouse tumor and depilation experiments. C57BL/6J mice (6- to 8-wk-old females) were obtained from The Jackson Laboratory (Bar Harbor, ME). Mice kept under pathogen-free conditions were used for tumor protection experiments at 8-12 wk of age. All mouse experiments were performed under protocols approved by the Institutional Animal Care and Utilization Committee of Memorial Sloan-Kettering Cancer Center according to National Institutes of Health animal care guidelines. C57BL/6J mice were immunized using particle bombardment once a week for $5 \mathrm{wk}$ with one of the following plasmids: hgp 75, mgp75, or null plasmid (WRG/BEN). Additional control animals were not immunized by the gene gun. 2 wk following the final immunization by the gene gun, C57BL/6J mice were injected intravenously through the tail vein with $2 \times 10^{5} \mathrm{~B} 16 \mathrm{~F} 10 / \mathrm{LM} 3$ melanoma cells in sterile normal saline (18). Mice were sacrificed at 16-22 d after tumor challenge, and surface lung metastases were scored and counted as black nodules under a dissecting microscope $(21,22)$. Surface lung metastases were detected by day 6-8 under a dissecting microscope and by day $8-12$ by eye. Statistical analysis of tumor growth was performed using the two-sided Student's $t$ test, assuming unequal variances and Wilcoxon scores (rank sums).

$T$ cell subsets were depleted in vivo by intraperitoneal administration of a rat mAb GK1.5 (anti-CD4; IgG2b) and mouse mAb 2.43 (anti-CD8; IgG2b). Both were produced as ascites from hybridomas received from the American Type Culture Collection. Depletion experiments were performed as described previously (18). Briefly, animals were injected intraperitoneally with $\mathrm{mAb}(0.25 \mathrm{ml}$ ascites fluid $)$ $3 \mathrm{~d}$ before tumor challenge and every $4 \mathrm{~d}$ thereafter. Antibodies depleted their respective $\mathrm{T}$ cell populations by $>95 \%$ as revealed by flow assisted cell sorting analysis. An additional group of animals did not undergo antibody depletion. Natural killer (NK) cell depletion was performed using mAb PK136 (anti-NK-1.1) (American Type Culture Collection). Antibodies ( $0.2 \mathrm{ml}$ of ascites fluid) were injected intraperitoneally $3 \mathrm{~d}$ before tumor challenge and every $4 \mathrm{~d}$ thereafter. Depletion of NK cells was assessed by $4 \mathrm{~h}{ }^{51} \mathrm{Cr}$-release assays with $5 \times$ $10^{3}$ YAC cells as targets and spleen cells as effector cells at effector/ target ratios of 100:1, 50:1, and 25:1. Depletion was shown to completely abrogate detectable NK activity. FcR $\gamma$ chain knockout $\left(\mathrm{FcR}^{-1-}\right)$ mice and wild-type $\left(\mathrm{FcR}^{+/+}\right)$controls have been described $(23,24)$, and $\mathrm{FcR}^{-1-}$ mice had been backcrossed to $\mathrm{C} 57 \mathrm{BL} / 6$ mice for more than eight generations (24). CD4 $4^{-1-}$ mice on a C57BL/6 background were obtained from The Jackson Laboratory (25). Depigmentation experiments were performed as described (18). $1 \mathrm{wk}$ following the final immunization, C57BL/6J mice were either shaved or shaved followed by depilation with Nair over the posterior flank and observed for coat color changes upon regrowth of hairs, following mice for 6-8 wk.

Immunoprecipitation, Western blot, and isotype analysis. Metabolic labeling and immunoprecipitation were performed as previously described $(26,27)$. Confluent B16F10/LM3 cells were metabolically labeled for $4 \mathrm{~h}$ with $300 \mu \mathrm{Ci}$ of $\left[{ }^{35} \mathrm{~S}\right]$ methionine $\left(\mathrm{EXPRE}^{35} \mathrm{~S}^{35} \mathrm{~S}\right.$ protein labeling mix; New England Nuclear DuPont, Boston, MA) per milliliter of medium per $4-5 \times 10^{6}$ cells. Cells were then washed with cold PBS and lysed in lysis buffer containing 1\% NP-40, 0.5\% deoxycholate, $10 \mathrm{mM}$ Tris $\mathrm{HCl}, \mathrm{pH} 7.5$, and $150 \mathrm{mM} \mathrm{NaCl}$ and fresh protease inhibitors added as described (26). Incorporation of $\left[{ }^{35} \mathrm{~S}\right]$ methionine into protein was measured by TCA precipitation. For each immunoprecipitate, $3 \times 10^{6} \mathrm{cpm}$ TCA-insoluble precipitate in $150 \mu \mathrm{l}$ of lysis buffer was incubated with mouse sera or control mouse mAb antibody TA99 against mouse and human gp75 (28), followed by the addition of $40 \mu \mathrm{l}$ of protein A-Sepharose (Pierce Chemical Co., Rockford, IL). Endo- $\beta$-N-acetylglucosaminidase H (Endo H) and $\mathrm{N}$-glycanase digestions were performed as previously reported (26). Immunoprecipitates were then washed five times with $10 \mathrm{mM}$ Tris $\mathrm{HCl}, \mathrm{pH} 7.5,150 \mathrm{mM} \mathrm{NaCl}, 5 \mathrm{mM}$ EDTA, and 1\% NP-40 (TNEN), five times with $1 / 10$ strength TNEN containing additional $0.5 \mathrm{M}$ $\mathrm{NaCl}$, and finally with distilled water. Proteins were analyzed by $9 \%$ SDS-polyacrylamide gel electrophoresis (SDS-PAGE) under reducing conditions. Radioactive protein was visualized by fluorography BioMax MR-2 film (Kodak, Rochester, NY) using Amplify (Amersham, Arlington Heights, IL).

For immunoprecipitation-Western blot, B16F10/LM3 melanoma cells were lysed followed by immunoprecipitation with mAb TA99 as described above. Immunoprecipitates were run on $8 \%$ SDS-PAGE under reducing conditions and transferred to Immobilon membrane (Millipore, Bedford, MA). The membranes were blocked overnight at $4^{\circ} \mathrm{C}$ in $5 \%$ non-fat dry milk, washed, and probed with mouse sera or preimmune mouse sera at 1:60 dilution in PBS0.2\% Tween-20. Blots were then washed and incubated with goat anti-mouse IgGhorseradish peroxidase and detected by an ECL kit from Amersham. For isotyping experiments, the second antibodies utilized in immunoprecipitation-Western experiments were goat anti-mouse alkaline phosphatase conjugates specific for mouse immunoglobulin isotypes IgG1, IgG2a, IgG2b, IgG3, and IgM (Fisher, Springfield, NJ). Detection used 5-bromo-4-chloro-3-indolyl phosphate and 4-nitro blue tetrazolium chloride substrates (Boehringer-Mannheim, Indianapolis, IN) in diethanolamine buffer, $\mathrm{pH} 9.6$.

\section{Results}

Genetic immunization with xenogeneic but not syngeneic gp75 DNA induces autoantibodies. The mouse brown locus glycoprotein gp75 (also known as TRP-1) is a differentiation antigen expressed by melanocytes and melanomas of C57BL/6 mice. We have previously observed that C57BL/6 mice are tolerant to self gp75, based on immunization with gp $75^{+}$cells, purified syngeneic gp 75, or syngeneic gp75 peptides plus various adjuvants and cytokines (21; data not shown). To investigate further immunity against a tumor differentiation antigen, we used DNA immunization with mouse gp75 (mgp75) or human gp75 (hgp75) DNA to assess whether syngeneic or xenogeneic DNA would induce evidence of autoimmunity. Mouse and human gp75 are highly conserved at the protein level, with $82 \%$ identity and $87 \%$ homology (6).

C57BL/6J mice were genetically immunized with DNA encoding full-length syngeneic $m g p 75$ or $h g p 75$ under the control of a cytomegalovirus promoter using particle bombardment. 
Mice were immunized once a week for $5 \mathrm{wk}$. Sera from these mice were assessed for autoantibodies against syngeneic gp75 by immunoprecipitation of lysates from metabolically labeled, syngeneic B16F10/LM3 melanoma cells. No mouse ( 0 out of 39 mice) immunized with syngeneic $m g p 75$ DNA had detectable antibodies to gp75. However, almost all C57BL/6J mice genetically immunized with $h g p 75$ developed autoantibodies to syngeneic gp75 (69 out of 71 mice). A representative experiment from 11 experiments is shown in Fig. 1.

Characterization of autoantibodies against syngeneic gp75. Autoantibodies against syngeneic gp75 in C57BL/6J appeared after the third immunization, although weakly (see Fig. 2), and peaked after the fourth and fifth immunization. Following the fifth immunization, autoantibodies against gp75 could still be detected by immunoprecipitation for as long as 6 mo without further boosts, showing that long-lived responses could be elicited, either due to memory or possibly to continued stimulation from self gp75. The autoantibodies against gp75 all recognized a mature glycosylated form of gp75 (with an apparent molecular mass of 73-78 kD) with a minor lower band of 66$68 \mathrm{kD}$ also recognized by sera from approximately half of the mice (Fig. 1). Both bands represented glycosylated forms of gp75, with the higher molecular weight band representing a mature, processed gp75 and the lower band representing a more immature glycosylated form of gp75. Both the higher and lower bands were digested to a $55 \mathrm{kD}$ core protein by $\mathrm{N}$-glycanase but were resistant to digestion by Endo $\mathrm{H}$ (data not shown), confirming their identity as gp75 with Asn-linked glycans that had been processed through the Golgi complex.

Antibodies against gp75 were IgG subclass, specifically $\mathrm{IgG} 1$ and $\mathrm{IgG} 2 \mathrm{~b}$ isotype with little or no $\mathrm{IgG} 2 \mathrm{a}$ response in five of five mice immunized with hgp75 (data not shown). The development of $\operatorname{IgG}$ antibodies suggested that antibody responses were dependent on $\mathrm{T}$ cell help. Sera from five $\mathrm{CD} 4^{-/-}$ mice immunized with hgp75 demonstrated weak or no antibody responses to gp75 by immunoprecipitation (data not

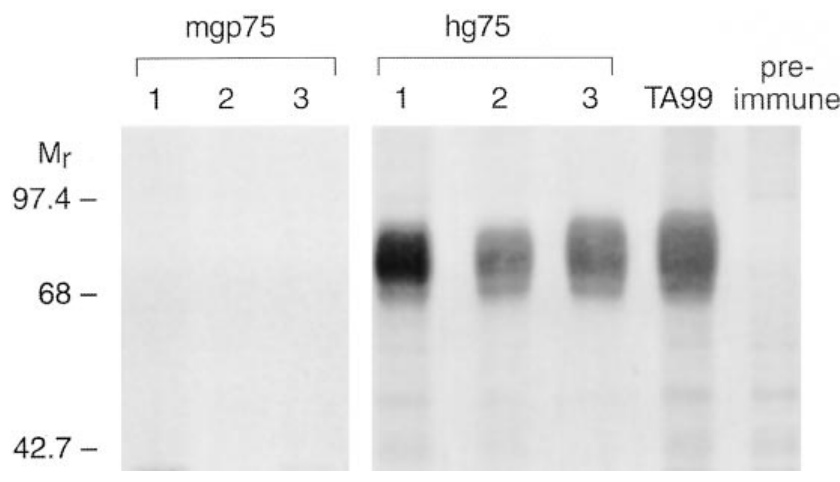

Figure 1. C57BL/6J mice immunized with human gp75 cDNA (hgp75), but not with syngeneic mouse gp75 cDNA (mgp75), produced antibodies against syngeneic mouse gp75. C57BL/6J mice were immunized weekly for $5 \mathrm{wk}$ with cDNA by particle bombardment. Representative results from mice immunized with $h g p 75$ and $\operatorname{mgp} 75(n=3$ per group) in the same experiment are shown. Sera from immunized mice were used to immunoprecipitate syngeneic gp 75 from lysates of syngeneic B16F10/LM3 melanoma cells metabolically labeled with $\left[{ }^{35}\right.$ S]methionine. The mAb TA99 was used as a positive control (TA99 lane). Preimmune serum was used as a control (preimmune lane). Immunoprecipitates were resolved by SDS-PAGE on a $9 \%$ gel. Apparent molecular mass markers are shown in the left lane $\left(M_{\mathrm{r}}\right)$. shown). These results showed that strong antibody responses were dependent on CD4-dependent recognition, presumably involving Th2-type help based on isotype of antibody responses.

Priming with hg75 breaks tolerance to immunization with mgp75. To determine whether immunization with hgp75 broke tolerance to syngeneic gp75 in C57BL/6J mice, we primed mice with $h g p 75$ for two immunizations followed by a third immunization with either $h g p 75, \operatorname{mgp} 75$, or null vector (eight mice per group). After two immunizations, autoantibodies were either not evident or barely detectable in mice immunized with $h g p 75$. After priming with $h g p 75$, a single DNA immunization with mgp75 generated strong antibody responses against syngeneic gp75 (Fig. 2) in seven out of eight mice. Only weak antibody responses were observed after the third immunization with hgp 75 (the three strongest responses are shown in Fig. 2) in only four of eight mice, as observed in our other experiments. These results show that priming with hgp75 did not simply generate cross-reactive antibodies against mouse gp75. Rather, priming with human gp75 broke tolerance to mouse gp75, allowing mouse gp75 to boost substantially $\mathrm{IgG}$ autoantibodies against self gp75.

Xenogeneic human gp75 DNA immunization induces protection to tumor challenge. The in vivo response after induction of autoantibodies to gp75 was investigated using a syngeneic tumor model. The gp75 glycoprotein is expressed in melanocytes and B16F10/LM3 melanoma cells of C57BL/6J mice. C57BL/6J mice that were immunized with hgp75 once a week for 5 wk were protected from lung metastases generated by B16F10/LM3 melanoma cells injected intravenously $2 \mathrm{wk}$ after the last immunization. Results of a representative experiment are shown in Fig. 3. In four separate experiments, C57BL/6J mice immunized with hgp75 ( $n=46$ total mice) were significantly protected from lung metastases (mean $41 \pm 15$ SD metastases when results were combined from all four experiments) compared with control mice (mean 292 \pm 15 metastases

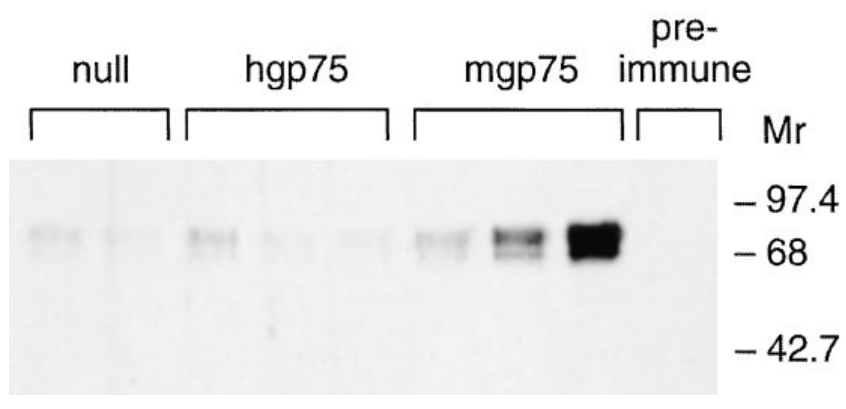

Figure 2. Immunization of C57BL/6J mice with human gp 75 cDNA (hgp75) breaks tolerance to immunization with syngeneic mouse gp75 cDNA (mgp75). Mice were immunized with hgp 75 twice $7 \mathrm{~d}$ apart. $1 \mathrm{wk}$ after the second immunization with $h g p 75$, mice were immunized a third time with either $h g p 75, \operatorname{mgp} 75$, or control null vector $W R G / B E N$. Representative results from mice immunized with hgp 75, $m g p 75$, and null ( $n=2-3$ mice/group) in the same experiment are shown. Preimmune serum was used as a control (preimmune lane). B16F10/LM3 melanoma cells were lysed followed by immunoprecipitation with mAb TA99. Immunoprecipitates were run on $8 \%$ SDS-PAGE under reducing conditions and transferred to Immobilon membrane, and the membranes were probed with sera from immunized mice or preimmune sera at 1:60 dilution. Apparent molecular mass markers are shown in the right lane $\left(M_{\mathrm{r}}\right)$. 


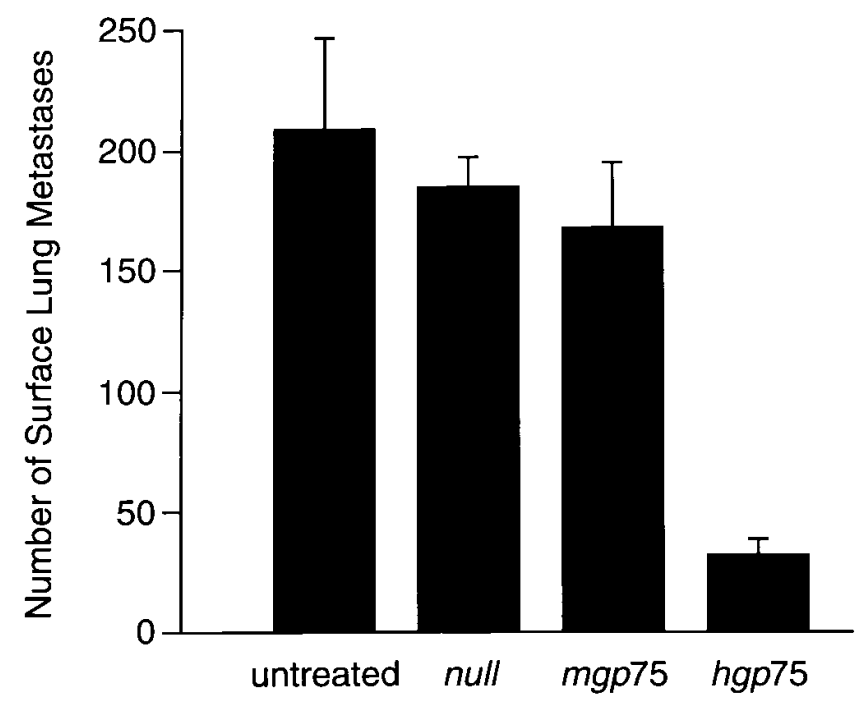

Figure 3. Tumor protection against B16F10/LM3 lung metastases was evaluated in mice genetically immunized with human gp75 (hgp75), syngeneic mouse gp75 (mgp75), or control null (WRG/BEN vector) cDNA. C57BL/6J mice were immunized by particle bombardment with cDNA once a week for $5 \mathrm{wk}$. 2 wk after the last immunization, mice were challenged through the tail vein with $2 \times 10^{5} \mathrm{~B} 16 \mathrm{~F} 10 /$ LM3 melanoma cells. One group of untreated mice was also challenged with melanoma cells (untreated). Surface metastatic lung nodules were scored $21 \mathrm{~d}$ after tumor challenge. There were 10-12 mice in each group. Data are expressed as mean \pm SD.

in 42 mice immunized with control null vector and mean $307 \pm 20$ metastases in 36 untreated mice for all experiments, $P>0.45)$. The difference between lung metastases in mice immunized with hgp75 compared with mice immunized with a null vector was highly significant $(P<0.0001)$, representing an $86 \%$ decrease in lung nodules. No significant protection was observed in mice immunized with syngeneic mgp 75 alone (Fig. 3 ). Thus, xenogeneic but not syngeneic gp75 protected against colonization of the lung by metastatic melanoma cells.

Mice immunized with hgp75 develop depigmentation. Mice were assessed for autoimmune manifestations by observing coat color. Animals were depilated or shaved after the last immunization, and the coat was allowed to regrow. Melanocytes in mice are present in the epithelium surrounding the hair bulb, and depilation removes hair at the bulb leading to stimulation of new hair growth cycles with deposition of pigment from melanocytes. Shaving does not effectively remove hair bulbs. C57BL/6J mice immunized with hgp75 developed depigmentation in previously depilated coats (12 out of 15 mice), while no depigmentation was observed in mice immunized with mgp75 (0 out of 15 mice) over 6 mo of observation (see Fig. 4). In mice that were depilated, depigmentation appeared as confluent white areas against a black coat background; this depigmentation was indistinguishable from coat color changes that were previously reported after passive immunization of C57BL/6J mice with mAb TA99 (18). In mice that were shaved but not depilated, depigmentation appeared as "salt and pepper" mixtures of depigmented and dark brown hairs in five out of five mice (Fig. 4). The difference in the appearance of depigmentation between depilated and shaved mice may reflect the more uniform stimulation of a new hair growth cycle and melanocyte activation triggered by depilation. In both de-

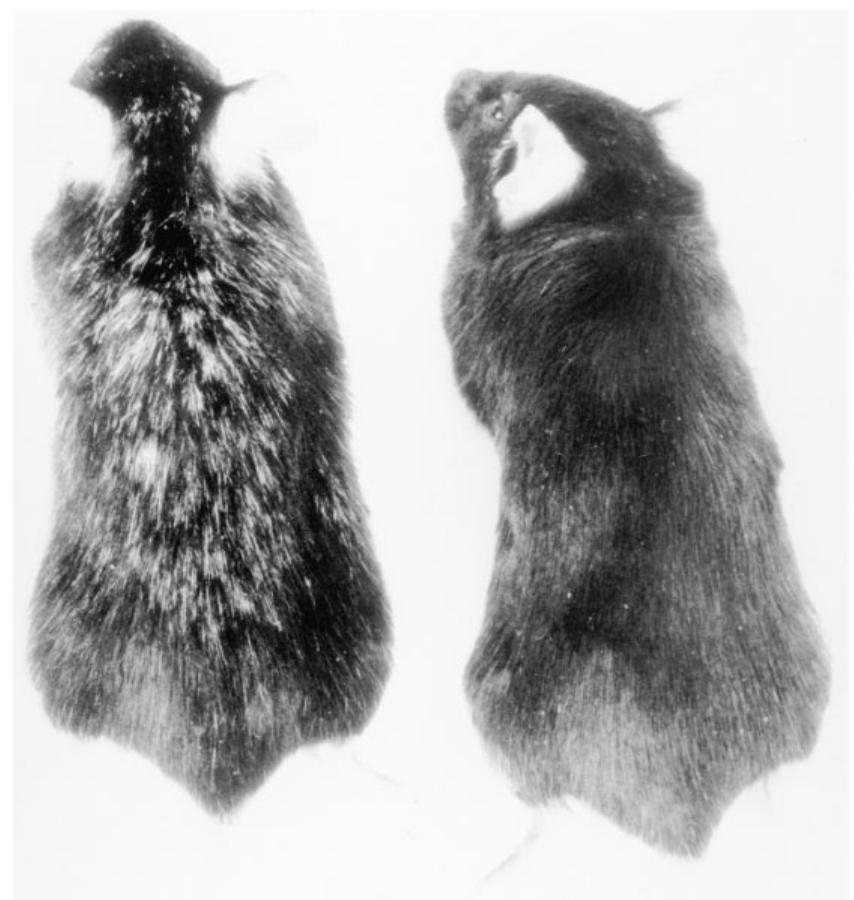

Figure 4. Depigmentation was observed in mice immunized with hgp 75 but not $m g p 75$ or null vector. C57BL/6J mice were immunized by particle bombardment with DNA once a week for $5 \mathrm{wk}$. After the final immunization, the dorsal aspect of the trunk was shaved with or without depilation and the coats were allowed to grow in naturally. After 3-4 wk, depigmentation was observed in coats of mice immunized with $h g p 75$ but not in mice immunized with either syngeneic mgp 75 or null vector. Depigmentation appeared as patchy white areas with map-like borders against a black coat in mice that were shaved and depilated (data not shown; see reference 18) or as a "salt and pepper" appearance in mice shaved without depilation (left mouse). A representative mouse genetically immunized with syngeneic mgp 75 did not show depigmentation after shaving (right mouse).

pilated and shaved mice, depigmentation was first noticed 3-4 wk after the last immunization with hgp 75 (approximately 40 $50 \mathrm{~d}$ after starting immunization). No changes in feeding habits, behavior, or general well-being were noted.

$\mathrm{CD}^{+}$and $\mathrm{NK}_{1.1^{+}}$cells and Fc $\gamma$ receptors play a role in tumor protection but not depigmentation following immunization with hgp75 DNA. To assess what components of the immune system might be involved in tumor rejection and depigmentation, T lymphocyte subsets or NK cell populations were depleted before challenge with tumor cells. Depletion of $\mathrm{CD}^{+}$ cells did not alter tumor rejection (mean $32 \pm 19$ metastases) mediated by immunization with hgp 75 (Fig. 5). Depletion of $\mathrm{CD}^{+}$cells partially reversed rejection of B16F10/LM3 lung metastases (mean $127 \pm 24$ metastases) by DNA immunization, showing that $\mathrm{CD}^{+}$cells played a role at the effector phase of tumor protection. A role for $\mathrm{CD}^{+}$cells was also detected in $\mathrm{CD}^{-/-}$mice (mean $174 \pm 35$ metastases), presumably due to the requirement for $\mathrm{CD}^{+} \mathrm{T}$ cell help in generating sufficient $\mathrm{IgG}$ antibody responses to gp75 as well as a potential role at the effector stage (Fig. 5).

Depletion of an NK1.1 $1^{+}$cell population reversed the protective effect of immunization with hgp75, supporting a role for NK cells in tumor protection in this model. C57BL/6J mice 


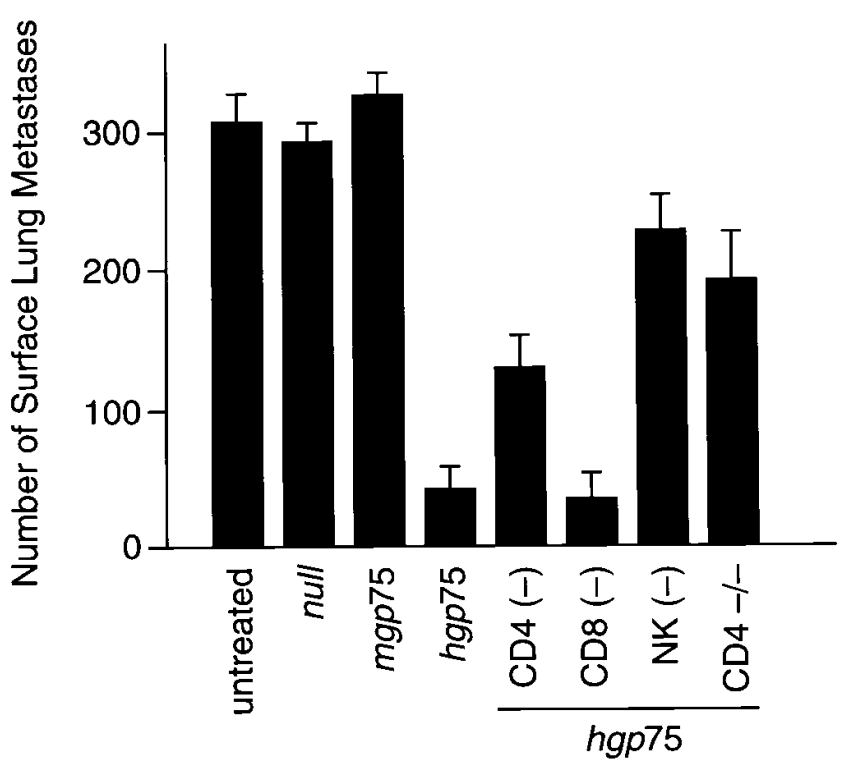

Figure 5. Potential effector functions involved in protection against B16F10/LM3 lung metastases were evaluated in mice genetically immunized with human gp75 (hgp75), mouse gp75 (mgp75), or control null (WRG/BEN vector) cDNA. C57BL/6J or $\mathrm{CD} 4^{-1-}$ mice were immunized by particle bombardment with cDNA once a week for $5 \mathrm{wk}$. 2 wk after the last immunization, mice were injected through the tail vein with $2 \times 10^{5} \mathrm{~B} 16 \mathrm{~F} 10 / \mathrm{LM} 3$ melanoma cells. One group of untreated mice were also challenged with melanoma (untreated). On day 21 after tumor challenge, surface metastatic lung lesions were scored. There were 10 mice in the untreated group, 9 mice in the null and $m g p 75$ groups each, and a total of 24 mice in the $h g p 75$ group (4-6/subgroup). Depletion of $\mathrm{CD} 4^{+}$or $\mathrm{CD} 8^{+} \mathrm{T}$ cell subsets in vivo was accomplished by intraperitoneal administration of mAb GK1.5 (anti-CD4) or mAb 2.43 (anti-CD8), respectively. NK cell depletion was performed using mAb PK136 (anti-NK1.1). A requirement for CD4 molecule at either the immunization or effector stage was also assessed by looking for tumor rejection in $\mathrm{CD}^{-/-}$mice. Data are expressed as mean $\pm \mathrm{SD}$.

depleted of NK1.1 ${ }^{+}$cells had no detectable protection from lung metastases (mean 225 \pm 27 metastases) compared with protection observed in control undepleted mice (mean $41 \pm 15$ metastases) (Fig. 5). Activation of Fc receptors I and/or III was implicated in tumor rejection using mice that were deficient in the $\gamma$ chain of these receptors. Fc $\gamma \mathrm{R}^{-1-}$ mice demonstrated no protection against tumor challenge after immunization with hgp75 (Fig. 6), indicating that activation of the $\gamma$ chain acting through FcRI and/or III was essential for development of autoantibodies and/or effector function in this model.

Depigmentation developed in all mice immunized with hgp75 after depletion of $\mathrm{CD}^{+}, \mathrm{CD}^{+}$, and NK1.1 $1^{+}$cells $(n=$ 3-4 mice/group). One could argue that antibody depletion of potential immune or effector cells was not uniform in blood and tissues due to biodistribution of the antibodies. Therefore, the distinction between tumor immunity and autoimmunity could be due to tissue distribution of antibodies, leading to incomplete depletion of lymphoid cells in skin. However, depigmentation also readily developed in all $\mathrm{CD}^{-1-}$ mice and in $\mathrm{FcR} \gamma^{-1-}$ mice ( $n=3$ mice/group), although tumor protection was decreased or abrogated in these strains of mice (Figs. 5 and 6). These results show that $\mathrm{CD}^{+}$cells and $\mathrm{Fc} \gamma$ receptors

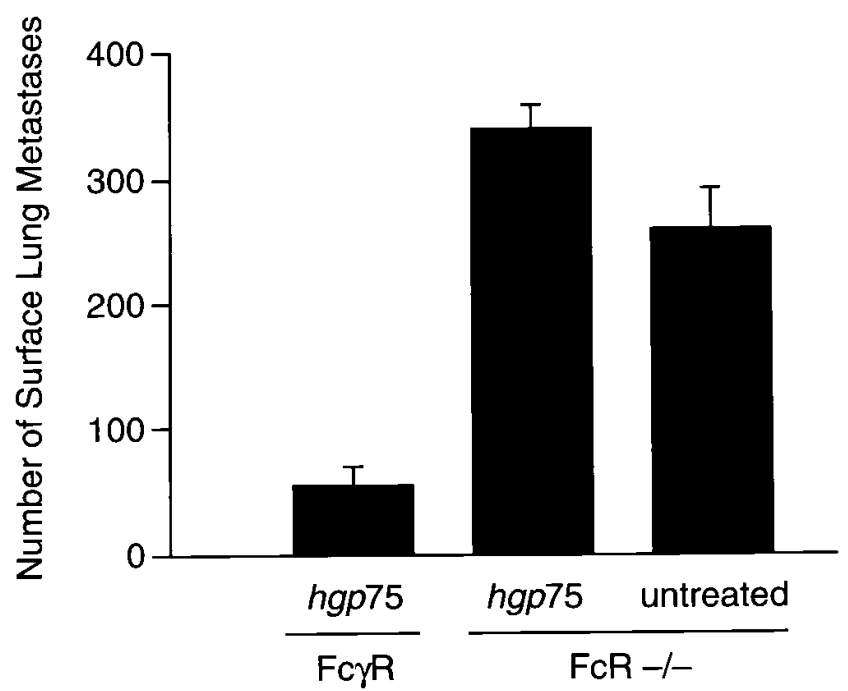

Figure 6. A study of Fc $\gamma$ chain receptors involved in protection against B16F10/LM3 lung metastases was performed in mice genetically immunized with human gp75 (hgp75). $\mathrm{Fc} \mathrm{R}^{-/-}$mice background $\left(\mathrm{Fc} \gamma \mathrm{R}^{-/-}\right)$or control $\mathrm{Fc} \gamma \mathrm{R}^{+/+}$mice $(\mathrm{Fc} \gamma \mathrm{R})$ were immunized with $h g p 75$ cDNA once a week for 5 wk. One group of untreated mice was challenged with melanoma (untreated). 2 wk after the last immunization, mice were injected through the tail vein with $2 \times 10^{5}$ B16F10/LM3 melanoma cells and scored as described in Fig. 5. On day 21 after tumor challenge, surface metastatic lung lesions were scored. There were five mice per group. Data are expressed as mean \pm SD.

are required for tumor protection but not autoimmune manifestations.

\section{Discussion}

Immune recognition of melanoma by antibodies and $\mathrm{T}$ cells has been intensely studied over the past two decades. Three categories of antigens have been defined from these studies. The first and most gratifying antigens are encoded by mutations in genes that are implicated in the pathogenesis and progression of melanoma. These include mutations in $C D K 4$, a cell cycle regulatory gene, and $\beta$-catenin, which participates in cell adhesion and growth $(29,30)$. Recognition of these mutant proteins suggests that the immune system can recognize altered gene products that participate in the malignant phenotype, but it is not clear how frequently this occurs. Only a small handful of these antigens has been identified in human cancers, each in only an individual patient. A second group of antigens is expressed by some melanomas and other cancers and by few normal tissues, particularly testes, but not normal tissue counterparts of the cancer. Examples include the MAGE antigens and related families $(31,32)$. This group has been termed "tumor-specific shared antigens," "cancer-testes antigens," or "oncospermatogonal antigens" $(32,33)$. None of these terms is particularly satisfying, and this reflects how little we know about the function of these antigens.

The third group is the differentiation antigens. The melanocyte differentiation antigens are the most prevalent antigens recognized on melanomas. The prototypes are the melanosome membrane glycoproteins, which include the tyrosinase family (tyrosinase, gp75, and TRP-2) along with the distantly 
related silver locus protein, gp100. The Melan A/MART-1 antigen is a fifth member whose intracellular localization and function are unknown (34). Differentiation antigens have been recognized by antibodies, cytotoxic $\mathrm{CD}^{+} \mathrm{T}$ cells, and $\mathrm{CD} 4^{+} \mathrm{T}$ cells of melanoma patients (6-16). The "cancer-testes" antigens have been recognized by antibodies and cytotoxic $\mathrm{CD} 8^{+}$ $\mathrm{T}$ cells $(31,35)$ and mutant proteins have been recognized so far by cytotoxic $\mathrm{CD} 8^{+} \mathrm{T}$ cells $(29,30)$.

Both $\mathrm{CD}^{+}$cytotoxic and $\mathrm{CD} 4^{+}$helper $\mathrm{T}$ cells from melanoma patients have been shown to recognize epitopes in tyrosinase, and autoantibodies against tyrosinase have been identified in patients with vitiligo and melanoma (7-12). Three points can be made. First, the immune repertoire contains B cells and cytotoxic and helper $\mathrm{T}$ cells that can potentially recognize tyrosinase family antigens. Second, as our knowledge of the immune repertoire against melanoma expands, the distinctions between specificity of antibody and $\mathrm{T}$ cell recognition of antigens become increasingly blurred. In fact, presumably the detection of high avidity antibodies reflects helper $\mathrm{T}$ cell responses, linking humoral and cellular immunity. Third, individual melanoma patients can recognize multiple antigens. An example is patient AV, studied at Memorial Sloan-Kettering, the University of Mainz, and at the Ludwig Institute for Cancer Research, Brussels, who had T cells in his repertoire that recognized tyrosinase, Melan-A/MART-1, and mutant CDK4, along with antibodies that recognized an undefined shared melanoma antigen (29). This patient had an extraordinary recovery with long-term survival after extensive regional metastatic melanoma.

We have chosen to focus on the most prevalent antigens on melanoma, the differentiation antigens. Recognition of these antigens presents many problems. First, how is recognition of these antigens possible in the face of immune tolerance? Second, even when recognition is possible, can this generate immunity that can reject cancer? Finally, if immunity to differentiation antigens can reject cancer, what are the potential autoimmune sequelae? Our studies in mice using the gp75 melanocyte differentiation antigen show that immunity, measured as tumor protection, can provide significant tumor protection. This is also associated with depigmentation, an autoimmune sequela that demonstrates concerns about toxicity when immunizing cancer patients against self antigens. The potential clinical implications of toxicity will depend on the normal tissue expression of the autoantigen, the sequelae of autoimmunity in that tissue, measured against the potential benefits for treating a person with cancer.

It is presumed that antibodies play a crucial role in this immunity because the presence of these autoantibodies corresponded to induction of tumor protection and our previous studies showed that passive transfer of a pure $\mathrm{IgG}$ antibody against gp75 could mediate rejection of tumors (18). However, other components of the immune system must play a role in the immunization and effector phases of tumor rejection, including $\mathrm{NK} 1.1^{+}$and $\mathrm{CD} 4^{+}$cells. In a previous report, C57BL/6 mice immunized with either cell-associated or purified forms of syngeneic gp75 protein combined with a variety of immune adjuvants and cytokines did not produce autoantibodies or cytotoxic T cells (CTL) to syngeneic gp75 (21). C57BL/6 are tolerant to syngeneic gp75 for both antibody and cytotoxic $\mathrm{T}$ cell responses, based on these studies plus additional experiments immunizing with purified gp75 and peptides from gp75 that bind $\mathrm{K}^{\mathrm{b}}$ (Moroi, Y., and A.N. Houghton, unpublished data).
However, immunization with the human melanoma cell line SK-MEL-19, which expresses xenogeneic gp75, and Freund's adjuvant did induce autoantibodies against syngeneic mouse gp75 in C57BL/6 mice with subsequent immunity (21). It was not possible in these studies to determine whether immunity to mouse melanoma and autoimmunity were specifically caused by immune recognition of human gp75, or whether immunity to other antigens shared by human and mouse melanoma contributed to tumor immunity and autoimmunity, or whether other cell-derived factors contributed to immunity. The present experiments show that immune recognition of human gp75 directly induced cross-reactive immunity to mouse gp75. More important, antibody recognition of human gp75 triggered a break in B or $\mathrm{T}$ cell tolerance to self gp 75 . We speculate that minor differences in protein sequences between human and mouse gp75 elicit T cell help, possibly by providing MHC class II restricted peptides in the xenogeneic gp75 that bind $\mathrm{MHC}$ class II with higher affinity. $\mathrm{CD}^{+} \mathrm{T}$ cells, probably Th2-type, participated in generating autoantibodies against self gp75. However, $\mathrm{CD}^{+}$cells also are linked to the effector phase of tumor rejection, so they may have a dual role both in priming immune responses and at the effector phase.

From the perspective of cancer immunology, the immune system is potentially replete with antibodies and $\mathrm{T}$ cells that can recognize antigens shared by cancer cells and normal cells. Thus, editing of the immune repertoire is not terribly stringent from the viewpoint of a cancer cell. Tolerance to melanoma in part occurs in the periphery, after selection during lymphocyte development, although "apparent" tolerance due to ignorance is also likely. Autoreactive T and B cells with high-affinity receptors against melanocyte antigens may be deleted during development, particularly if they are exposed to these antigens in the thymus or bone marrow. Immune cells with intermediate or low-affinity receptors may remain in the repertoire, and these lymphocytes either infrequently respond to melanoma antigens or are triggered by antigen mimicry or cross-reactivity. Cross-reactivity is based on homologies between an evolutionarily conserved inciting antigen and the ultimate target antigen. Our study shows that a highly homologous protein can trigger autoantibodies to a self protein shared by melanoma and melanocytes. The tyrosinase family of proteins, including gp75/tyrosinase-related protein 1 , has been highly conserved throughout vertebrate evolution, including primates, rodents, birds, and amphibians. The scenario of breaking tolerance against melanoma differentiation antigens is not restricted to antibody responses nor to just cross-reactivity between proteins conserved through evolution. Loftus and colleagues suggest that cytotoxic $\mathrm{CD}^{+} \mathrm{T}$ cells against a peptide in the Melan A/MART-1 melanocyte differentiation antigen, which is shared with melanoma, might be provoked by exposure to viruses or bacteria that express homologous peptides (36). Candidates for peptide mimicry of Melan-A/MART-1 included a peptide derived from glycoprotein $\mathrm{C}$ of the prevalent viral pathogen HSV-1. This would provide one explanation for the observation that precursor cytotoxic $\mathrm{T}$ cells against Melan A/MART-1 can be readily isolated from both melanoma patients and healthy individuals (37). With regard to our studies using DNA immunization, an extreme speculation is that homologous DNA in the environment might trigger cross-reactive immunity, e.g., autoimmunity, if the DNA were inadvertently taken up and expressed by antigen-presenting cells. In any case, recognition of homologous proteins or peptides, ei- 
ther conserved through evolution or unrelated, can trigger autoimmunity and immunity to cancer.

\section{Acknowledgments}

We are grateful for discussions and help from Michael Feldman, Rubin Dyall, Isao Hara, and Yoshizumi Takechi. We thank PowderJect, Inc. for providing the gene gun apparatus used for particle bombardment to deliver DNA.

This work was supported by NCI grant RO1 CA56821, Swim Across America, The Louis and Anne Abrons Foundation, The Share Foundation, and the Milstein Family Fund. J. Wolchok is the first recipient of the H-C Fellowship Award, and was supported in part by grants NCI CA09512 and CA09207.

\section{References}

1. Pardoll, D.M. 1994. Tumour antigens. A new look for the 1990s. Nature. 369:357.

2. Houghton, A.N. 1994. Cancer antigens: immune recognition of self and altered self. J. Exp. Med. 180:1-4.

3. Boyse, E.A., and L.J. Old. 1969. Some aspects of normal and abnormal cell surface genetics. Annu. Rev. Genet. 3:269-290.

4. Houghton, A.N., M. Eisinger, A.P. Albino, J.G. Cairncross, and L.J. Old. 1982. Surface antigens of melanocytes and melanomas. Markers of melanocyte differentiation and melanoma subsets. J. Exp. Med. 156:1755-1766.

5. Hearing, V.J., and K. Tsukamoto. 1991. Enzymatic control of pigmentation in mammals. FASEB J. 5:2902-2909.

6. Vijayasaradhi, S., B. Bouchard, and A.N. Houghton. 1990. The melanoma antigen gp75 is the human homologue of the mouse $b$ (brown) locus gene product. J. Exp. Med. 171:1375-1380.

7. Brichard, V., A. Van Pel, T. Wolfel, C. Wolfel, E. De Plaen, B. Lethe, P. Coulie, and T. Boon. 1993. The tyrosinase gene codes for an antigen recognized by autologous cytolytic T lymphocytes on HLA-A2 melanomas. J. Exp. Med. 178:489-495.

8. Robbins, P.F., M. El-Gamil, Y. Kawakami, E. Stevens, J.R. Yannelli, and S.A. Rosenberg. 1994. Recognition of tyrosinase by tumor-infiltrating lymphocytes from a patient responding to immunotherapy. Cancer Res. 54:3124-3126.

9. Topalian, S.L., L. Rivoltini, M. Mancini, N.R. Markus, P.F. Robbins, Y. Kawakami, and S.A. Rosenberg. 1994. Human CD4 ${ }^{+}$T cells specifically recognize a shared melanoma-associated antigen encoded by the tyrosinase gene. Proc. Natl. Acad. Sci. USA. 91:9461-9465.

10. Song, Y.-H., E. Connor, Y. Li, B. Zorovich, P. Balducci, and N. Maclaren. 1994. The role of tyrosinase in autoimmune vitiligo. Lancet. 344:10491052.

11. Merimsky, O., E. Baharav, Y. Shoenfeld, S. Chaitchik, R. Tsigelman, D. Cohen-Aloro, and P. Fishman. 1996. Anti-tyrosinase antibodies in malignant melanoma. Cancer Immunol. Immunother. 42:297-302.

12. Kemp, E.H., D.J. Gawkrodger, S. MacNeil, P.F. Watson, and A.P. Weetman. 1997. Detection of tyrosinase autoantibodies in patients with vitiligo using ${ }^{35} \mathrm{~S}$-labeled recombinant human tyrosinase in a radioimmunoassay. $J$. Invest. Dermatol. 109:69-73.

13. Wang, R.-F., P.F. Robbins, Y. Kawakami, X.-Q. Kang, and S.A. Rosenberg. 1995. Identification of a gene encoding a melanoma tumor antigen recognized by HLA-A31-restricted tumor-infiltrating lymphocytes. J. Exp. Med. 181: 799-804.

14. Wang, R.-F., E. Appella, Y. Kawakami, X. Kang, and S.A. Rosenberg. 1996. Identification of TRP-2 as a human tumor antigen recognized by cytotoxic T lymphocytes. J. Exp. Med. 184:2207-2216.

15. Kemp, E.H., D.J. Gawkrodger, P.F. Watson, and A.P. Weetman. 1997. Immunoprecipitation of melanogenic enzyme autoantigens with vitiligo sera: evidence for cross-reactive autoantibodies to tyrosinase and tyrosinase-related protein-2 (TRP-2). Clin. Exp. Immunol. 109:495-500.

16. Bakker, A.B., M.W. Schreurs, A.J. de Boer, Y. Kawakami, S.A. Rosenberg, G.J. Adema, and C.G. Figdor. 1994. Melanocyte lineage-specific antigen gp100 is recognized by melanoma-derived tumor-infiltrating lymphocytes. $J$. Exp. Med. 179:1005-1009.

17. Fidler, I.J. 1973. Selection of successive tumour lines for metastasis. Nat.
New Biol. 242:148-149.

18. Hara, I., Y. Takechi, and A.N. Houghton. 1995. Implicating a role for immune recognition of self in tumor rejection: passive immunization against the brown locus protein. J. Exp. Med. 182:1609-1614.

19. Shibahara, S., Y. Tomita, T. Sakakura, C. Nager, B. Chaudhuri, and R. Muller. 1986. Cloning and expression of cDNA encoding mouse tyrosinase. $\mathrm{Nu}$ cleic Acid Res. 14:2413-2427.

20. Ross, H., L. Weber, S. Wang, G. Piskun, R. Dyall, P. Song, Y. Takechi, J. Nikolic-Zugic, A.N. Houghton, and J.L. Lewis. 1997. Priming for T cell-mediated rejection of established tumors by cutaneous DNA immunization. Clin. Cancer Res. 3:2191-2196.

21. Naftzger, C., K. Takechi, H. Kohda, H. Hara, S. Vijayasaradhi, and A.N. Houghton. 1996. Immune response to a differentiation antigen induced by altered antigen: a study of tumor rejection and autoimmunity. Proc. Natl. Acad. Sci. USA. 93:14809-14814.

22. Hara, I., H. Nguyen, Y. Takechi, B. Gansbacher, P.B. Chapman, and A.N. Houghton. 1995. Rejection of mouse melanoma elicited by local secretion of interleukin-2: implicating macrophages without $\mathrm{T}$ cells or natural killer cells in tumor rejection. Int. J. Cancer. 61:253-260.

23. Sylvestre, D.L., and J.V. Ravetch. 1994. Fc receptors initiate the Arthus reaction: redefining the inflammatory cascade. Science. 265:1095-1098.

24. Clynes, R., Y. Takechi, Y. Moroi, A. Houghton, and J.V. Ravetch. 1998. Fc receptors are required in passive and active immunity to melanoma. Proc. Natl. Acad. Sci. USA. 95:652-656.

25. Rahemtulla, A., W.-P. Fung-Leung, M. Schilham, T. Kundig, S. Sambhara, A. Narendran, A. Arabian, A. Wakeham, C. Paige, T. Zinkernagel, et al. 1991. Mice lacking CD4 have normal development and function of CD $8^{+}$cells but have markedly decreased helper cell activity. Nature. 353:180-184

26. Vijayasaradhi, S., Y. Xu, B. Bouchard, and A.N. Houghton. 1995. Intracellular sorting and targeting of melanosomal membrane proteins: identification of signals for sorting of the human brown locus protein, gp75. J. Cell Biol. 130:807-820.

27. Xu, Y., V. Setaluri, Y. Takechi, and A.N. Houghton. 1997. Sorting and secretion of a melanosome membrane protein, gp75/TRP1. J. Invest. Dermatol. 109:788-795.

28. Thomson, T.M., F.X. Real, S. Murakami, C. Cordon-Cardo, L.J. Old, and A.N. Houghton. 1988. Differentiation antigens of melanocytes and melanoma: analysis of melanosome and cell surface markers of human pigmented cells with monoclonal antibodies. J. Invest. Dermatol. 90:459-466.

29. Wolfel, T., M. Hauer, J. Schneider, M. Serrano, C. Wolfel, E. Klehmann-Hieb, E. De Plaen, T. Hankeln, K.H. Meyer zum Buschenfelde, and D Beach. 1995. A p16INK4a-insensitive CDK4 mutant targeted by cytolytic T lymphocytes in a human melanoma. Science. 269:1281-1284.

30. Robbins, P., M. El-Gamil, Y. Li, Y. Kawakami, D. Loftus, E. Appella, and S. Rosenberg. 1996. A mutated beta-catenin gene encodes a melanomaspecific antigen recognized by tumor infiltrating lymphocytes. J. Exp. Med. 183: 1185-1192.

31. van der Bruggen, P., C. Traversari, P. Chomez, C. Lurquin, E. De Plaen, B. Van den Eynde, A. Knuth, and T. Boon. 1991. A gene encoding an antigen recognized by cytolytic T lymphocytes on a human melanoma. Science. 254: $1643-1647$.

32. Old, L.J., and Y.T. Chen. 1998. New paths in human cancer serology. J. Exp. Med. 187:1163-1167.

33. Boon, T., and L.J. Old. 1997. Cancer tumor antigens. Curr. Opin. Immunol. 9:681-683.

34. Kawakami, Y., S. Eliyahu, C.H. Delgado, P.F. Robbins, K. Sakaguchi, E. Appella, J.R. Yannelli, G.J. Adema, T. Miki, and S.A. Rosenberg. 1994 Identification of a human melanoma antigen recognized by tumor-infiltrating lymphocytes associated with in vivo tumor rejection. Proc. Natl. Acad. Sci. USA. 91:6458-6462.

35. Stockert, E., E. Jager, Y.T. Chen, M.J. Scanlan, I. Gout, J. Karbach, M. Arand, A. Knuth, and L.J. Old. 1998. A survey of the humoral immune response of cancer patients to a panel of human tumor antigens. J. Exp. Med. 187: 1349-1354.

36. Loftus, D.J., C. Castelli, T.M. Clay, P. Squarcina, F.M. Marincola, M.I. Nishimura, G. Parmiani, E. Appella, and L. Rivoltini. 1996. Identification of epitope mimics recognized by CTL reactive to the melanoma/melanocytederived peptide MART-1(27-35). J. Exp. Med. 184:647-657.

37. Marincola, F.M., L. Rivoltini, M.L. Salgaller, M. Player, and S.A. Rosenberg. 1996. Differential anti-MART-1/MelanA CTL activity in peripheral blood of HLA-A2 melanoma patients in comparison to healthy donors: evidence of in vivo priming by tumor cells. J. Immunother. Emphasis Tumor Immunol. 19:266-277. 